\title{
SPATIAL DISTRIBUTED CHARACTERISTICS OF DYNAMIC RESPONSE OF JACKET SUPPORTED OFFSHORE WIND TURBINE UNDER HURRICANE INDUCED ENVIRONMENTAL LOADS
}

\author{
Bowen Jiang ${ }^{1}$, Qingshan Yang ${ }^{1}$, Guoqing Huang ${ }^{1}$ and Min Liu ${ }^{1}$ \\ ${ }^{1}$ School of Civil Engineering, Chongqing University \\ No.174 Shazhengiie, Shapingba, Chongqing, China \\ jbw1991222@163.com
}

Keywords: Offshore wind turbines, Hurricane, Dynamic responses, Spatial distribution

\begin{abstract}
During extreme tropical cyclones such as hurricanes and typhoons, although offshore wind turbines (OWTs) are parked and feathered to mitigate loads on the turbine blades, failures may happen to OWTs due to hurricane induced environmental loads or errors of the control systems. In this study, attempts are made to investigate the spatial distributed characteristics of hurricane induced response of OWTs considering the influence of the misalignment between local wind and wave. The dynamic responses of the jacket supported OWT are computed via implementing coupled aero-servo-hydro elastic analysis based on the hurricane-related wind and wave fields. Parametric models are adopted to simulate the spatial dependent turbulent wind fields and uni-directional irregular wave fields induced by hurricanes. Based on a series of coupled aero-servo-hydro elastic analyses, the spatial distributed characteristics of dynamic response such as tower-top and platform displacement, structural stress in tower and substructure and axial force on cross-section of the legs are investigated by comparing their maximum, mean and standard deviation values. In addition, focusing on the points where maximum responses occur, the time history curves of response are obtained and analyzed to illustrate their temporal characteristics.
\end{abstract}




\section{INTRODUCTION}

As a widely concerned renewable energy, offshore wind energy undoubtedly provides a solution to the environmental problems encountered in the process of energy utilization. However, the harvest of wind resource is companied with potential risks due to extreme tropical cyclones such as typhoons and hurricanes. In southeast coastal area of China where many offshore wind farms are located, there is a high risk that OWTs are exposed by tropical cyclones. From 2003 to 2008, a number of wind turbine failure cases during tropical cyclone activities were reported and attracted the attention of some scholars [1-3]. These failure events all happened to onshore wind turbines in the Asia-Pacific region and can be characterize as structural failure and blade failure. According to Li's study [3], the cause of the failures during hurricane events are correlated with extreme wind speed, drastic change of wind direction, intense turbulence in the inflow wind and fail of control system. During hurricane events, extreme wind and waves are generated causing the bearing loads more complicated than that on onshore wind turbines, which may lead to failures of OWTs although there is no record of these events.

Based on the above considerations, environmental loads due to hurricanes should be considered in the design future turbines. One of the major problems in analyzing the characteristics of OWT response caused by hurricanes is modelling spatio-temporal correlated wind and wave fields. In the past few decades, some sophisticated numerical models such as WAVEWATCH III, UMCM and SWAN [4-6] have been used to predict the wind and wave within hurricanes based on the physics of wave generation. However, due to the high computational cost and limited resolution, these numerical models are not appreciable for the dynamic analysis of OWTs. To this end, some parametric models were proposed to characterize hurricane generated wind and waves. For hurricane wind field model, Holland model [7] has been extensively used to characterize the hurricane radial profiles due to its simplicity and effectiveness. Some other parametric models for hurricane winds have been proposed taking into account the effect of surface friction and storm translation [8-11]. Attempts have also been made to provide parametric wave models generated by hurricanes [12-19]. The spatial distributed characteristic of significant wave height and local wave directions are investigated in these works.

Inspired by the failure cases of wind turbines reported before (although these failure events all happened to onshore wind turbines), the control system, turbine parking strategy and blade azimuthal configuration have vital influence on the aerodynamic load of a wind turbine. Kim and Manuel [5] investigated the hurricane induced loads on a monopole supported 5-MW OWT with considerations for nacelle yaw and blade pitch control and validated the most unfavorable yaw angle during hurricanes. For OWTs supported by substructures such as jackets and tripods, the structural bearing capacities are different when being loaded in different directions. Wei et al [20] investigated the bearing capacities of a jacket supported OWT in different directions. The lowest loading capacity at $45^{\circ}$ is verified based on a static nonlinear pushover analysis of the jacket structure under wind and wave loads. Furthermore, attentions have been paid to hurricane risk assessment of OWTs and directional effects of environmental loads on OWTs [21-23].

In summary, the wind fields and wave fields induced by hurricanes are spatial distributed due to the characteristics of hurricane wind and physical mechanism of wave generation. Therefore both the amplitude and the direction of environmental loads vary with the spatial location. Despite the studies above, limited studies have been conducted on characteristics of hurricane induced dynamic response of jacket supported OWTs focusing on the spatial correlated wind and wave fields generated by hurricanes. 


\section{MODELING OF WIND AND WAVE INDUCED BY HURRICANES}

The dynamic responses of OWTs depend on the environmental loading which is remarkably correlated with the wind fields and wave fields induced by hurricanes. However, due to the movement and intensity variation of storm, the hurricane induced wind fields and wave fields evolve over time. Therefore, the wind speed or the wave elevation at any specified point can be modeled as non-stationary processes. Despite the time-varying characteristics, the wind speed or the wave elevation can be characterized by stationary processes during a short interval $(10 \mathrm{~min})$ based on the slow-change characteristic of hurricane wind fields. Thus, parametric models are adopted to simulate the spatial dependent wind field and wave field during hurricanes.

\subsection{Modeling of hurricane wind}

The near-surface hurricane wind field, namely the mean wind speed at height of $10 \mathrm{~m}$ can be decomposed into an axisymmetric component of the storm vortex and an axially asymmetric component of the surface background wind [11]. The schematic for calculation of the nearsurface hurricane wind at any specified point is shown in Figure 1. As is defined in Figure 1, storm track is assumed to be aligned with the horizontal axis and the storm center is fixed to the coordinate origin. $r$ and $\varphi$ are radial distance from storm center to observation point $P$ and azimuth of vector $O P$ which is defined counter-clockwise from the horizontal axis. The coordinate of the observation point $P$ is $\left(X_{P}, Y_{P}\right)$, where $Y_{P}$ represents the offset distance of point $P$ from the storm track; $X_{P}$ represents the horizontal distance of point $P$ from the storm center $O$. The near-surface hurricane wind speed equals the vector sum of the component of the storm vortex and the component of the surface background wind. Meanwhile, a deceleration effect and a rotational effect are caused by the surface friction therefore the gradient wind and background wind should be modified by reduction factor $\left(\alpha_{r}\right.$ and $\left.\alpha_{t}\right)$ and rotation angle $(\alpha$ and $\beta$ ).

$$
\vec{U}=\alpha_{r} \vec{V}_{r}+\alpha_{t} \vec{V}_{t}
$$

Where $\vec{U}$ is the mean wind speed at height of $10 \mathrm{~m} ; \alpha_{r}$ and $\alpha_{t}$ are surface wind reduction factor (SWRF) of gradient wind speed $V_{r}$ and background wind speed $V_{t}$.

In the present study, the gradient wind and the background wind are converted to the surface level with SWRF $\alpha_{r}=0.8$ and $\alpha_{t}=0.5$. The rotation angle of background wind $\beta=20^{\circ}$ while the rotation angle of gradient wind $\alpha$ is given by the National Weather Service's expression [8]

$$
\alpha=\left\{\begin{array}{l}
10^{\circ}\left(1+r / R_{m}\right), 0 \leq r<R_{m} \\
20^{\circ}+25^{\circ}\left(r / R_{m}-1\right), R_{m} \leq r<1.2 R_{m} \\
25^{\circ}, r \geq 1.2 R_{m}
\end{array}\right.
$$

Where $R_{m}$ is radius to maximum wind.

The value of the axisymmetric component of the storm vortex can be calculated based on the Holland model [7]. 


$$
V_{r}(r)=\left\{\left(\frac{R_{m}}{r}\right)^{B} \frac{100 B \Delta P \exp \left[-\left(\frac{R_{m}}{r}\right)^{B}\right]}{\rho}+\frac{r^{2} f_{c}^{2}}{4}\right\}^{0.5}-\frac{f_{c} r}{2}
$$

Where $\rho$ is air density; $f_{c}=2 \Omega \sin \Phi$ is the Coriolis parameter, in which $\Omega=7.292 \times 10^{-5}$ and $\Phi$ is lattice; $\Delta P$ is central pressure deficit; $B$ is the Holland parameter which is determined as

$$
B=\frac{V_{m}^{2} e \rho+f_{c} V_{m} R_{m} e \rho}{100 \Delta P}
$$

Where $e$ is base of natural logarithms; $V_{m}$ is maximum mean wind speed.

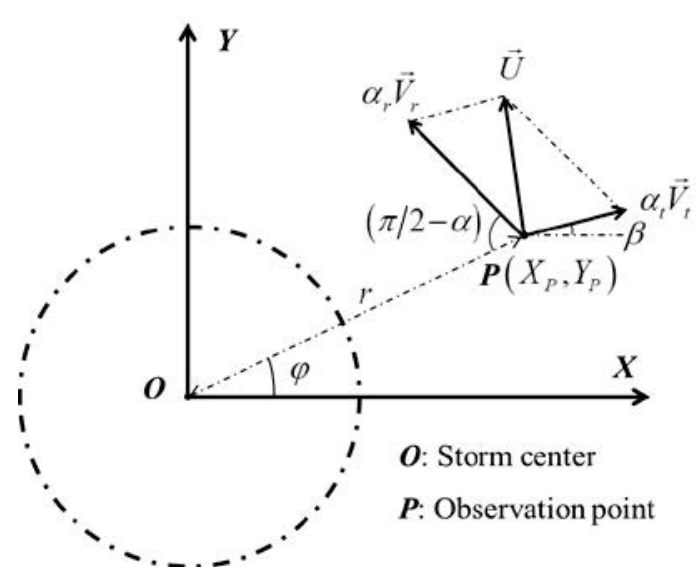

Figure 1: Schematic for calculation of the near-surface hurricane wind at any specified point

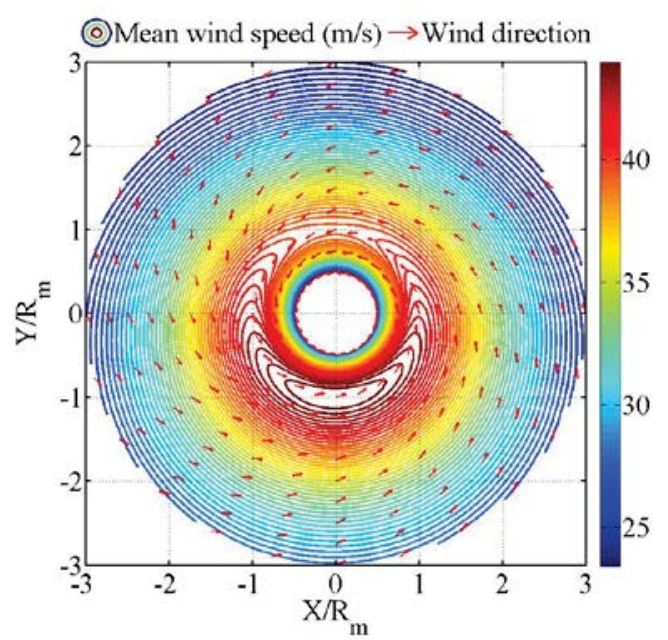

Figure 2: Contour of hurricane wind field at height of $10 \mathrm{~m}$

\begin{tabular}{cc}
\hline Parameters & values \\
\hline Latitude & $30.8 \mathrm{deg}$ \\
Storm central pressure deficit & $52 \mathrm{mb}$ \\
Radius to maximum winds & $74 \mathrm{~km}$ \\
Forward movement speed & $4.5 \mathrm{~m} / \mathrm{s}$ \\
Air density & $1.15 \mathrm{~kg} / \mathrm{m}^{3}$ \\
\hline
\end{tabular}

Table 1: Parameters of a measured hurricane

Using the hurricane wind model mentioned above, the spatial distribution of mean wind speed at height of $10 \mathrm{~m}$ can be simulated according to the parameters of a measured hurricane listed in Table 1. The contour of hurricane wind field at height of $10 \mathrm{~m}$ is shown in Figure 2. 
As is shown in Figure 2, due to the background wind, a peak occurs at the point where $r=R_{m}$ and $\varphi=270^{\circ}$.

\subsection{Modeling of hurricane wave}

In early studies, wind generated seas are characterized by wave spectra based on measured data which are related with the steady wind speed over the sea $[24,25]$. However, during meteorological forcing events such as tropical clones, hurricanes and typhoons, destructive waves are generated by time-varying wind fields correlated with the vortex structure. To this end, the concept of an extended fetch was proposed by Young [12-14] to reflect to the relationship between significant wave height, maximum wind velocity in the storm and velocity of the forward movement of the storm. More recently, fetch- or duration-limited wave growth functions [16-19] have been proposed according to the datasets from the National Aeronautics and Space Administration (NASA) and National Oceanic and Atmospheric Administration (NOAA) combined hurricane hunter missions during Bonnie 1998 [15, 26] and Ivan 2004 [27]. The fetch-limited wave growth function can be expressed as:

$$
\left\{\begin{array}{l}
H_{s}=8.10 \times 10^{-4} U_{10}^{1.19} x_{\eta}^{0.405} \\
T_{p}=9.28 \times 10^{-2} U_{10}^{0.526} x_{\omega}^{0.237}
\end{array}\right.
$$

Where $H_{s}$ is significant wave height; $T_{p}$ is spectral peak wave period; $U_{10}$ is mean wind speed at height of $10 \mathrm{~m} ; x_{\eta}$ and $x_{\omega}$ are effective fetch corresponding to $H_{s}$ and $T_{p}$ respectively.

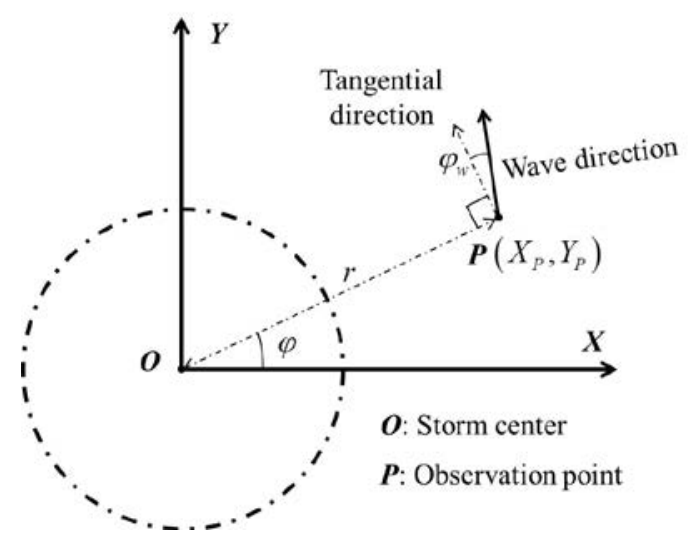

Figure 3: Schematic of azimuthal dependent wave direction

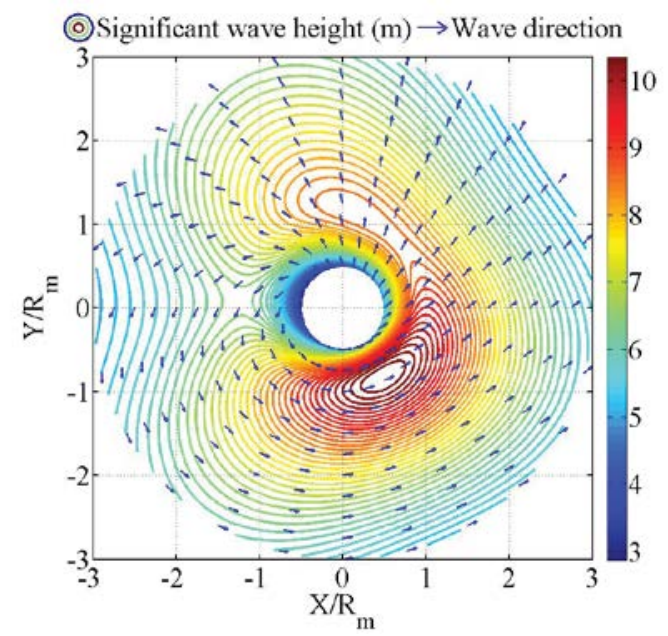

Figure 4: Contour of significant wave height

In this model, the variables $x_{\eta}$ and $x_{\omega}$ in fetch-limited wave growth function as well as the angle between wave direction and tangential direction $\varphi_{w}$ are azimuthal dependent as is shown in Figure 3. In Figure 3, the storm track is assumed to be aligned with the horizontal axis and the storm center is fixed to the coordinate origin. The detailed method to determine these variables can be found in Hwang's studies [18, 19]. In present study, Hwang's model was adopted to simulate hurricane wave field according to the hurricane wind field presented 
in Section 2.1. The spatial distributed significant wave height, spectral peak wave period and directions of wind and waves are shown in Figures 4-6.

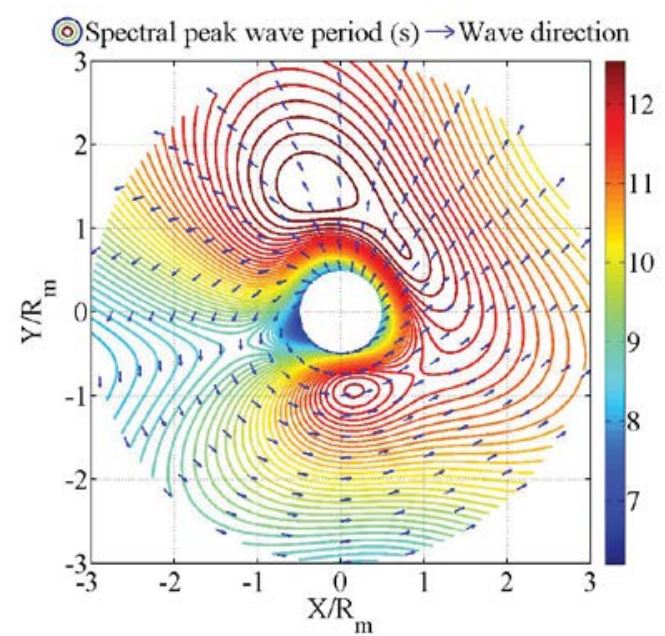

Figure 5: Contour of spectral peak wave period

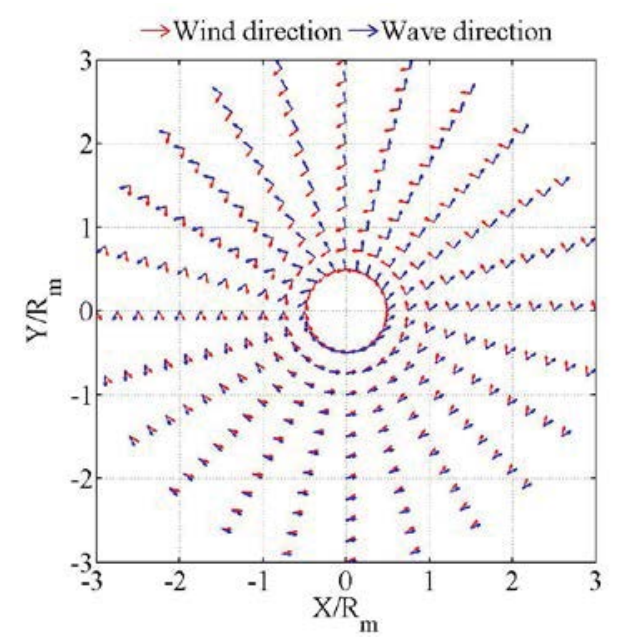

Figure 6: Azimuthal dependent wind and wave directions

\section{NUMERICAL SIMULATION}

\subsection{Modeling of OC4-jacket OWT}

In this study, a jacket design, namely OC4-jacket is considered to support the NREL 5MW baseline turbine [28, 29]. The main dimensions and properties of OC4-jacket and NREL 5-MW baseline turbine are listed in Table 2. The schematic of the OC4-jacket support structure is shown in Figure 7. As shown in Figure 7, the supporting structural system of OC4jacket is designed to be deployed in the site with 50-m water depth with the rotor-nacelle assembly (RNA) being mounted $90 \mathrm{~m}$ above mean sea level (MSL). In order to realize the rigid connection between tower and substructure, a rigid platform is employed. Moreover, the effect of nonlinear soil-pile interaction is neglected, in other words, the 4 legs of the jacket are assumed clamped at the seabed.

To simulate the parked wind turbine during hurricanes, the turbine blades were pitched to 90 and the RNA was yawed to be aligned with the local wind direction (shown in Figure 8). As a compromise of save computing cost, the wind turbine was assumed to be located within the range of $0.5 R_{m} \sim 3.0 R_{m}$ from the storm center. 


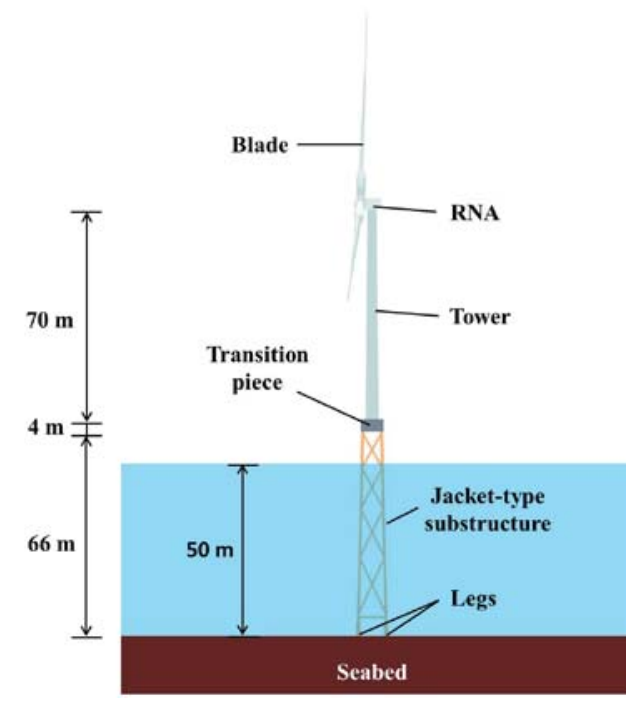

Figure 7: Schematic of an OC4-jacket OWT

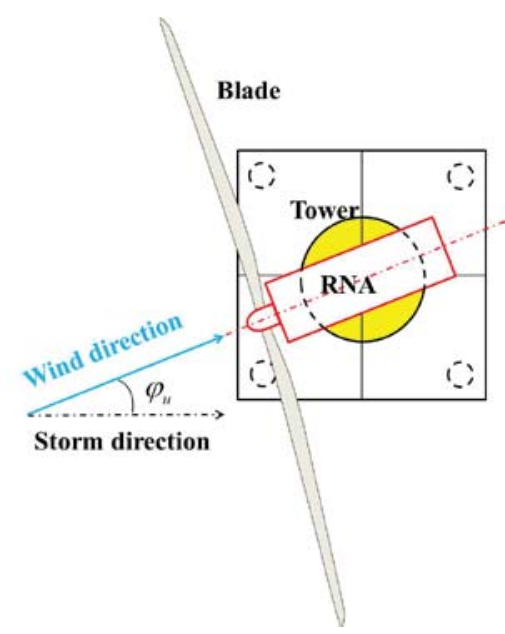

Figure 8: Schematic of nacelle yaw angle

\begin{tabular}{cc}
\hline Properties & values \\
\hline Rated power & $5 \mathrm{MW}$ \\
Cut in wind speed, rated wind speed, cut out wind speed & $3 \mathrm{~m} / \mathrm{s}, 11.3 \mathrm{~m} / \mathrm{s}, 25 \mathrm{~m} / \mathrm{s}$ \\
Rotor diameter, hub diameter & $126 \mathrm{~m}, 3 \mathrm{~m}$ \\
Hub height above MSL & $90 \mathrm{~m}$ \\
Deck height above MSL & $16 \mathrm{~m}$ \\
Tower length & $68 \mathrm{~m}$ \\
Transition-piece length & $4 \mathrm{~m}$ \\
Tower-base outer diameter & $6 \mathrm{~m}$ \\
Tower-base thickness & $0.032 \mathrm{~m}$ \\
Tower-top outer diameter & $4 \mathrm{~m}$ \\
Tower-top thickness & $0.03 \mathrm{~m}$ \\
RNA mass & $350000 \mathrm{~kg}$ \\
Jacket mass & $581256 \mathrm{~kg}$ \\
Transition piece mass & $666000 \mathrm{~kg}$ \\
Tower mass & $229812 \mathrm{~kg}$ \\
Structural damping ratio & $1 \%$ \\
\hline
\end{tabular}

Table 2: Main properties of OC4-jacket and NREL 5-MW baseline turbine 


\subsection{Environmental loads}

The inflow wind can cause aerodynamic load on OWTs including drag and lift force on blades and drag force on towers. With the hurricane wind field determined in Section 2.1, the Frøya wind profile and spectral model [30] were adopted to calculate the time series of inflow wind speed at different locations. The Frøya wind profile is expressed as

$$
\bar{u}(z)=U_{r e f}\left(1+0.0573 \sqrt{1+U_{\text {ref }}}\right) \ln \left(z / H_{\text {ref }}\right)
$$

Where $\bar{u}(z)$ is mean wind speed at height $z ; U_{\text {ref }}$ is the 1-hour mean wind speed at $H_{\text {ref }}$, $H_{r e f}=10 \mathrm{~m}$.

The Frøya spectral model was developed for neutral conditions over water in the Norwegian Sea. It can be expressed as

$$
S_{u}(f)=\frac{320\left(\frac{U_{r e f}}{10}\right)^{2}\left(\frac{z}{10}\right)^{0.45}}{\left(1+\tilde{f}^{n}\right)^{\frac{5}{3 n}}}
$$

Where $n=0.468$ and

$$
\tilde{f}=172 f\left(\frac{z}{10}\right)^{2 / 3}\left(\frac{U_{\text {ref }}}{10}\right)^{-0.75}
$$

In practice, blade element momentum (BEM) theory is one of most commonly used methods for calculating aerodynamic load on wind turbine blades. According to this method, twodimensional airfoil tables of lift and drag coefficient are included as a function of the angle of attack, thus a set of equations can be iteratively solved for the induced velocities on each blade element. For parked turbines, the rotor wake/induction effects can be ignored. Thus, the aerodynamic load on blades and tower can be directly calculated based on the tables of lift and drag coefficient and instantaneous inflow wind speed.

The hydrodynamic load on OWTs is related to local wave kinematics. The linear Airy wave theory [31] is used to predict the kinematics of two-dimensional waves of small height in deep water. In order to reflect the stochasticity of sea states, irregular waves can be modelled as the linear superposition of sinusoidal wave components. Thus, the distribution of wave energy at different frequencies is characterized by specifying a wave spectrum. For a developing sea, the JONSWAP (the Joint North Sea Wave Project) spectrum was proposed and adopted by IEC standard $[25,32]$. The IEC JONSWAP spectrum is expressed as

$$
S_{J S}(f)=0.3125 H_{s}^{2} T_{p}\left(\frac{f}{f_{p}}\right)^{-5} \exp \left[-1.25\left(\frac{f}{f_{p}}\right)^{-4}\right](1-0.287 \ln \gamma) \gamma^{\exp \left[-0.5\left(\frac{\frac{f}{f_{p}}-1}{\sigma}\right)^{2}\right]}
$$

Where $S_{J S}(f)$ is IEC JONSWAP spectrum; $f_{p}$ is spectral peak wave frequency, $f_{p}=1 / T_{p} ; \sigma=0.07$ for $f \leq f_{p}, \sigma=0.09$ for $f>f_{p} ; \gamma$ is peak-shape parameter: 


$$
\gamma\left\{\begin{array}{l}
5, \frac{T_{p}}{\sqrt{H_{s}}} \leq 3.6 \\
\exp \left(5.75-1.15 \frac{T_{p}}{\sqrt{H_{s}}}\right), 3.6<\frac{T_{p}}{\sqrt{H_{s}}} \leq 5 \\
1, \frac{T_{p}}{\sqrt{H_{s}}}>5
\end{array}\right.
$$

Based on the wave kinematics determined by JONSWAP spectrum as a function of significant wave height $H_{s}$ and spectral peak wave period $T_{p}$, the wave force on slender members can be calculated by Morison's equation [33]

$$
f_{H}=f_{D}+f_{I}=\frac{1}{2} C_{D} \rho_{w} D\left(u_{x}-\dot{x}\right)\left|u_{x}-\dot{x}\right|+C_{M} \rho_{w} \frac{\pi D^{2}}{4} \frac{\mathrm{d} u_{x}}{\mathrm{~d} t}-\left(C_{M}-1\right) \rho_{w} \frac{\pi D^{2}}{4} \ddot{x}
$$

Where $f_{H}$ is hydrodynamic force of a small size cylinder per unit length; $f_{I}$ and $f_{D}$ are the inertia force and drag force per unit length; $C_{M}$ and $C_{D}$ are the inertia coefficient and drag coefficient; $u_{x}$ is the horizontal velocity component of water particle; $\rho_{w}$ is water density; $D$ is the diameter of cylinder; $\mathrm{d} u_{x} / \mathrm{d} t$ is the horizontal acceleration component of water particle; $\dot{x}$ and $\ddot{x}$ are the horizontal velocity component and horizontal acceleration component of the cylinder.

\subsection{Dynamic analysis}

The structural response of OWTs subjected to simultaneous wind and wave is nonlinear and influenced by the interaction of aerodynamic, hydrodynamic, structural, operational, and geotechnical effects. The open-source software for turbine dynamic simulation, FAST [34], developed at National Renewable Energy Laboratory (NREL) are used in calculating the structural response of OWTs subjected to simultaneous wind and wave, where aerodynamic load and hydrodynamic load are obtained as described in previous steps. In this case, six 700$\mathrm{s}$ simulations were conducted assuming that the OWT is located in different positions within the range of $0.5 R_{m} \sim 3.0 R_{m}$ from the storm center with the first $100 \mathrm{~s}$ of dynamic responses removed.

Through numerical calculation, the maximum, mean, and standard deviation values of dynamic responses in key components were extracted. To reveal spatial distributed characteristics of dynamic response, the contour of maximum dynamic responses with respect to normalized spatial coordinates $X / R_{m}$ and $Y / R_{m}$ were obtained and analyzed.

Assuming that the storm intensity and the forward movement speed of the storm remains constant, the spatial distributed hurricane wind and wave field can be converted to time varying hurricane wind and wave field. As the storm track is aligned with the horizontal axis and the storm center is fixed to the coordinate origin, the observation point $P$ moves in the negative direction of the horizontal axis with an offset distance $Y_{P}$. Thus, the time series of mean wind speed, significant wave height and maximum value of dynamic response can be obtained by extracting points from contours of dynamic response on line $Y=Y_{P \max }$. It should be noticed that $Y_{P \max }$ is offset distance of the point where maximum response occurs (Fig 9 (a)). 


\section{RESULTS AND ANALYSIS}

\subsection{Tower-top and platform displacement}

Figure 9 shows contours of maximum, mean and standard deviation value of tower-top displacement and platform displacement. In Figs 9 (a-f), $d_{T \max }, d_{T \text { mean }}, d_{T S T D}, d_{P \max }$, $d_{P \text { mean }}$ and $d_{P S T D}$ stand for maximum tower-top displacement, mean tower-top displacement, standard deviation of tower-top displacement, maximum platform displacement, mean platform displacement and standard deviation of platform displacement respectively. As is shown in Figure 9 (c-d), being the reflection of hurricane wind field, $d_{T \text { mean }}$ and $d_{P \text { mean }}$ have almost the same characteristic in spatial distribution. In addition, $d_{T \max }, d_{T S T D}, d_{P \max }$, and $d_{P S T D}$ have similar characteristic in spatial distribution: the maximum value locates at the same point where $r=R_{m}$ and $\varphi=255^{\circ}$. The point of maximum displacement has an offset distance $Y_{P_{\max }}=-71.48$.This is mainly because aerodynamic load has dominant effect on tower-top displacement and platform displacement. Apart from the mean response determined by mean wind load, the azimuth of the maximum response is affected by dynamic response which is related to dynamic loads and structural resistance.

The time history of normalized mean wind speed $U_{10}$, significant wave height $H_{s}$ and maximum displacement $d_{\max }$ at the point of maximum displacement are shown in Figure 10. From Figure 10, the maximum displacement occurs near the eyewall after the wind speed reaches the peak value. In addition, similar to the time history curve of mean wind speed, the time history curve of maximum displacement shows unimodal characteristic. However, even if the aerodynamic load has dominant effect on tower-top displacement and platform displacement, the time history curve of maximum displacement is asymmetric while the time history curve of mean wind speed is of high symmetry due to the low forward movement speed of the storm. This phenomenon is correlated with non-axisymmetry of supporting substructure and temporal correlated wind-wave misalignment angle.

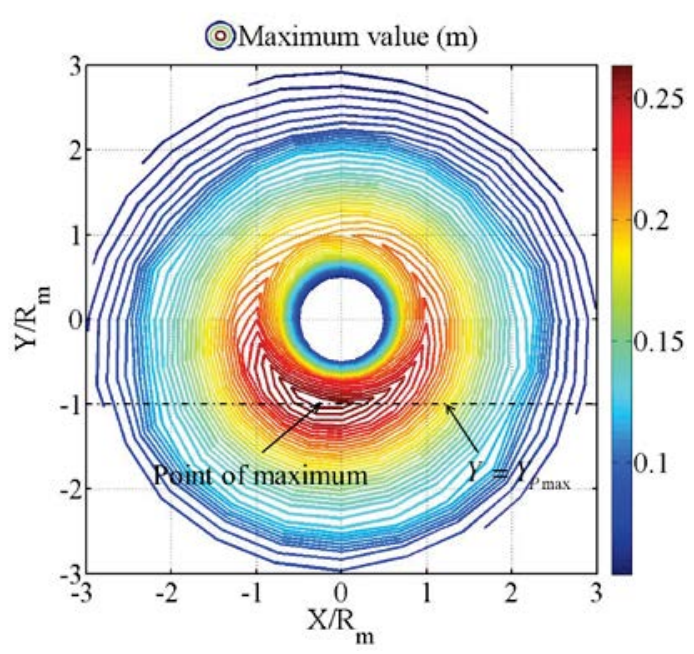

(a) $d_{T \max }$

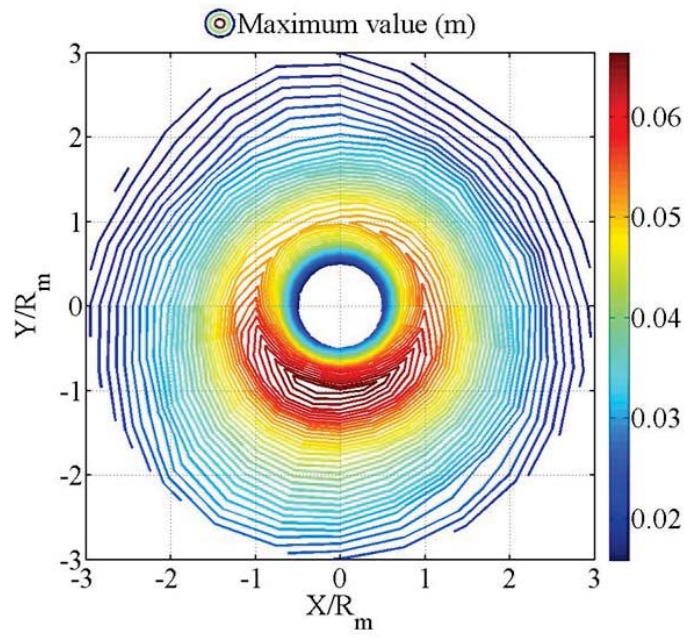

(b) $d_{P \max }$ 


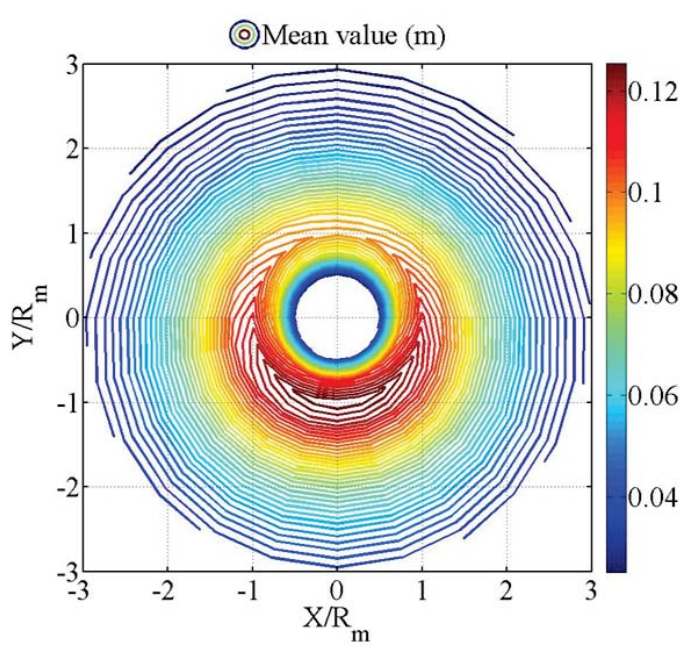

(c) $d_{T \text { mean }}$

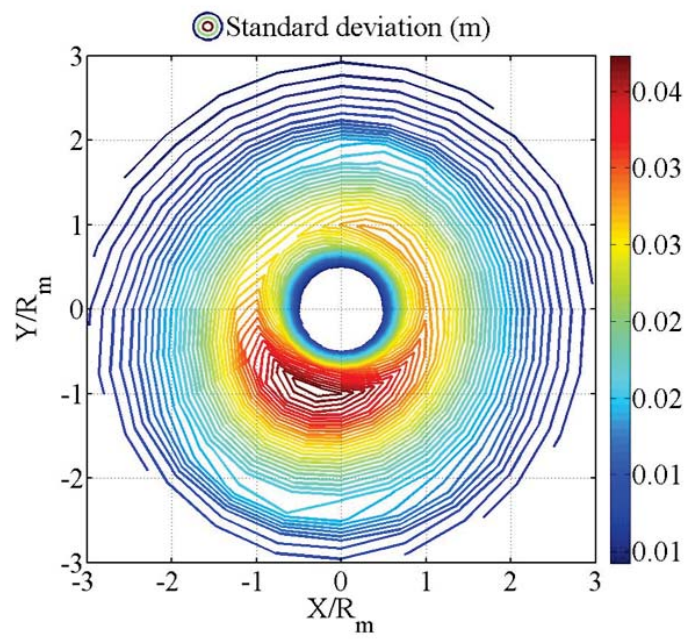

(e) $d_{T S T D}$

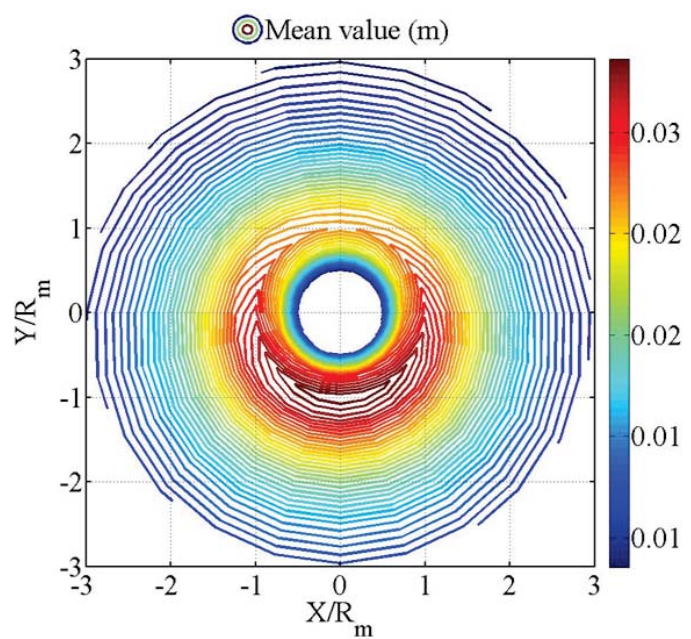

(d) $d_{P \text { mean }}$

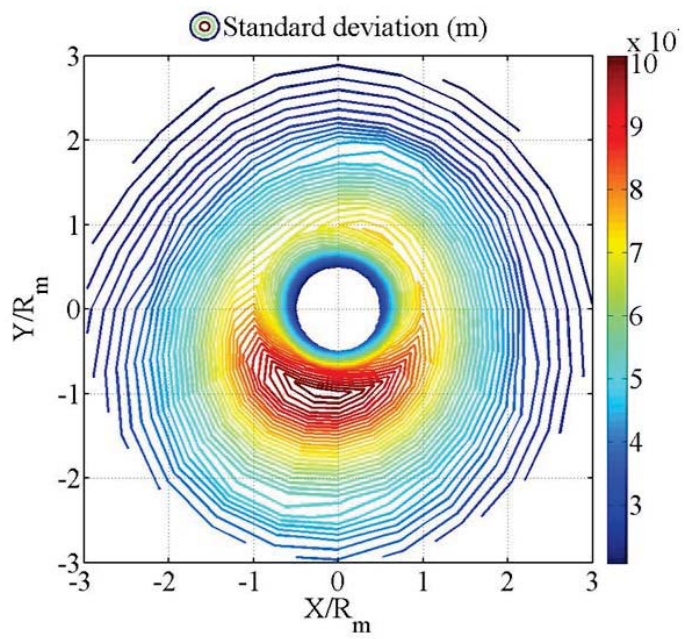

(f) $d_{P S T D}$

Figure 9: Contours of maximum, mean and standard deviation value of tower-top displacement and platform displacement 


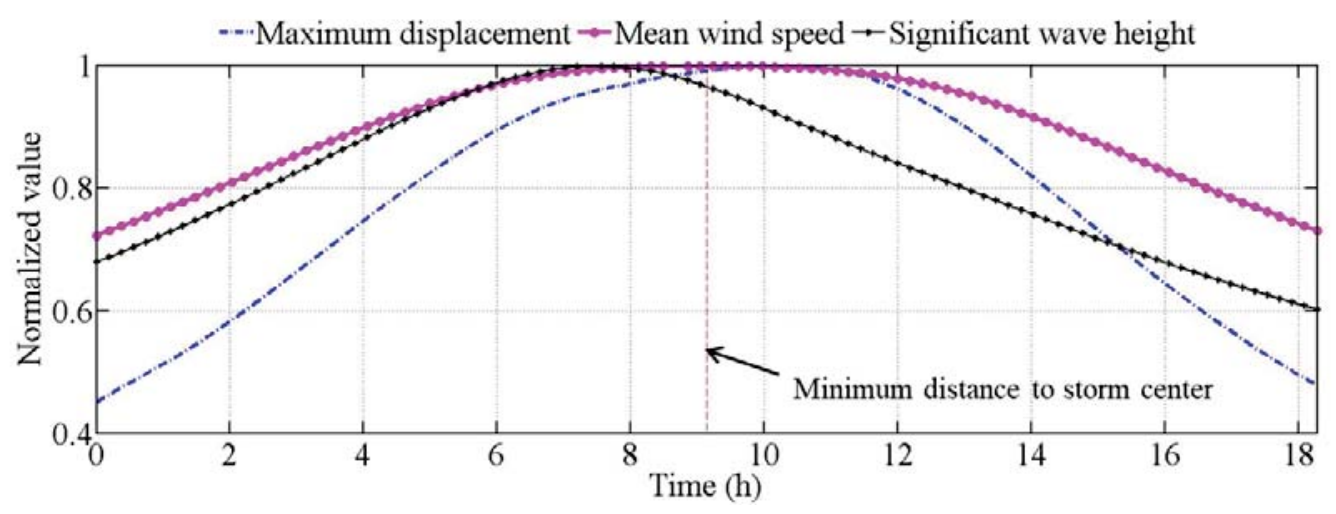

Figure 10: Time history of normalized mean wind speed, significant wave height and maximum displacement

\subsection{Stress in tower and substructure}

Contours of maximum normal stress and maximum shear stress in tower and substructure are shown in Figure 11 (a-d). In Figure 11 (a-d), $\sigma_{N T \max }, \sigma_{N J \max }, \sigma_{S T \text { max }}$ and $\sigma_{S J \max }$ stand for maximum tower normal stress, maximum normal stress in substructure, maximum tower shear stress, maximum shear stress in substructure respectively. From Figure 11 (a-d), the distribution characteristic of $\sigma_{N T \max }$ and $\sigma_{S T \text { max }}$ are similar to those of $d_{T \text { max }}$. However, $\sigma_{N J \max }$ and $\sigma_{S J \max }$ show different characteristic in spatial distribution. The maximum value of $\sigma_{N J \max }$ and $\sigma_{S J \max }$ can be found at the point where $r=R_{m}$ and $\varphi=285^{\circ}$. This point is almost coincident with the point where maximum significant wave height occurs. Meanwhile, another peak of $\sigma_{N J \max }$ and $\sigma_{S J \max }$ locates near the point where maximum value of $d_{T \max }$ occurs. Therefore, conclusion can be made that the maximum values of $\sigma_{N J \max }$ and $\sigma_{S J \max }$ should be attributed to hydrodynamic load while the maximum values of $\sigma_{N T \max }$ and $\sigma_{S T \text { max }}$ should be attributed to aerodynamic load.

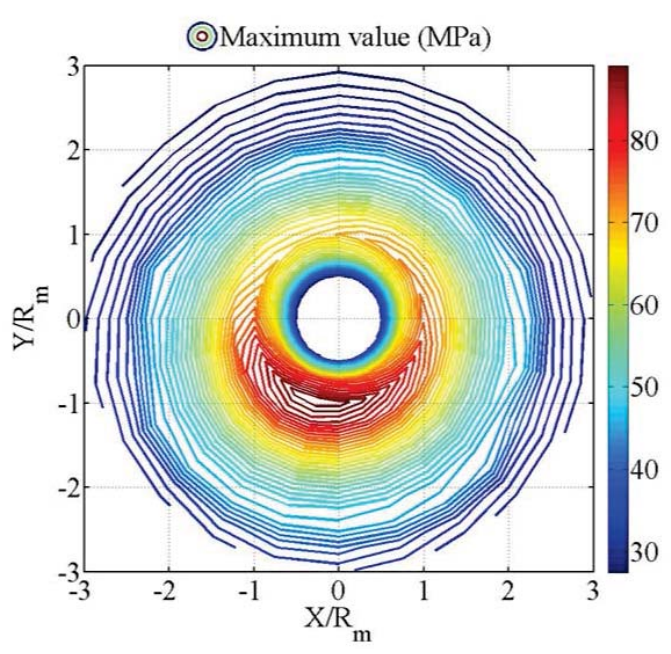

(a) $\sigma_{N T \max }$

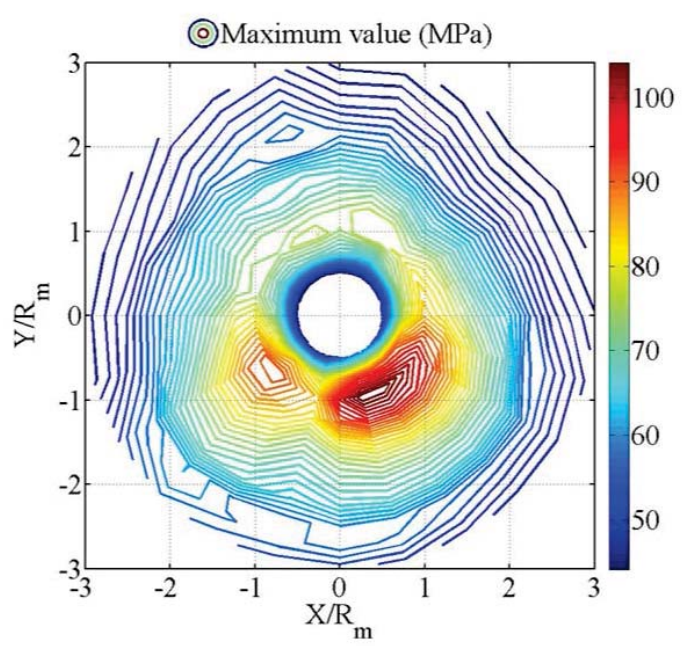

(b) $\sigma_{N J \max }$ 


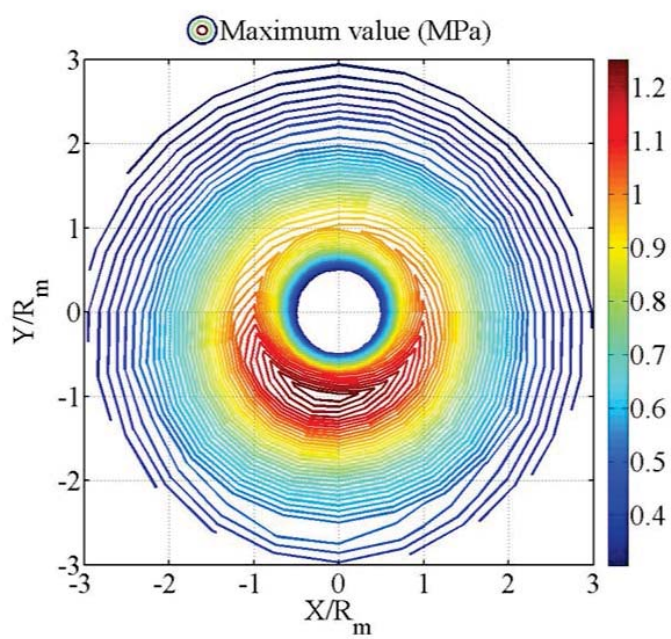

(c) $\sigma_{S T \max }$

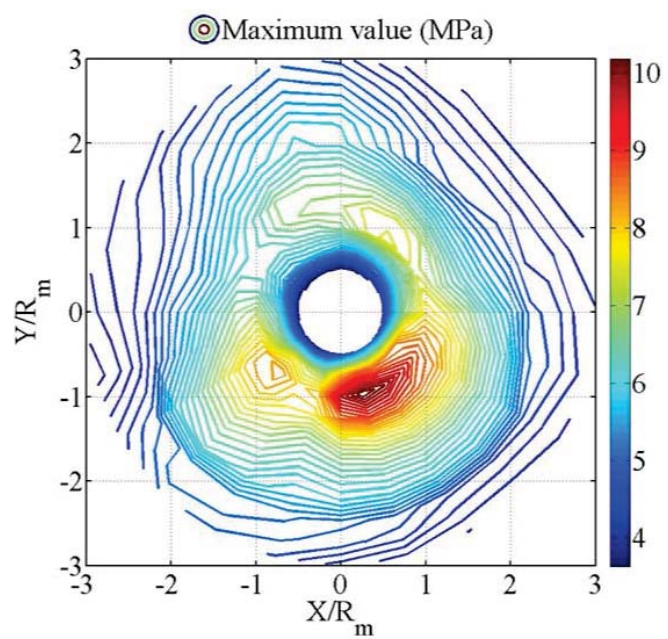

(d) $\sigma_{S J \max }$

Figure 11: Contours of maximum normal stress and maximum shear stress in tower and substructure

According to the same method above, we can obtain the time history curves of normalized maximum normal stress $\sigma_{N \max }$ and normalized maximum shear stress $\sigma_{S \max }$ at the points where maximum values occur (shown in Figures 12-13). It should be noted that the maximum normal stress and maximum shear stress do not locate at the same point. Thus, the time history curves of normalized mean wind speed $U_{10}$ and significant wave height $H_{s}$ at corresponding points are shown in Figures 12-13. As Figures 12-13 show, the time history curves of normalized maximum normal stress $\sigma_{N \text { max }}$ and normalized maximum shear stress $\sigma_{S \max }$ are bimodal with the maximum values occur before the arrival of minimum distance to storm center. With the intensity of hydrodynamic loads at their maximum values, the points of maximum response coincide with the points of maximum significant wave height.

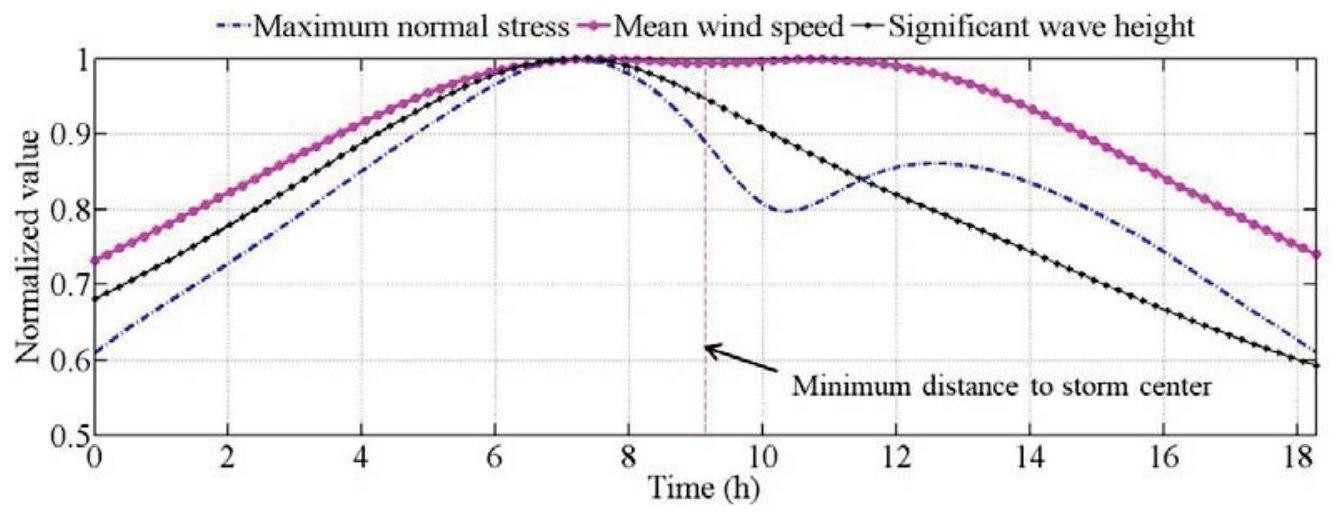

Figure 12: Time history of normalized mean wind speed, significant wave height and maximum normal stress 


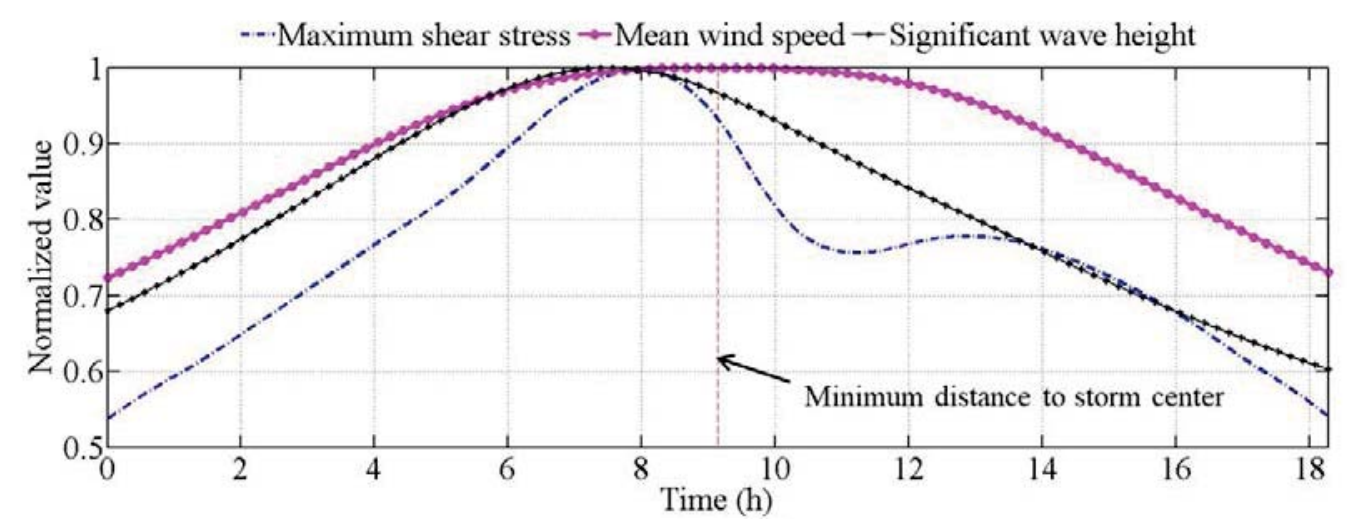

Figure 13: Time history of normalized mean wind speed, significant wave height and maximum shear stress

\subsection{Uplift and downward axial force on legs}

A part from the failure induced by excessive displacement and structural stress, the ultimate limit state of an OWT can also be determined by foundation uplift and yielding of underlying soil. Therefore, the contours of maximum uplift axial force $N_{U \max }$ and downward axial force $N_{D \max }$ on legs were checked (shown in Figure 14). From Figure $14(\mathrm{a}-\mathrm{b})$, the distribution characteristic of $N_{U \text { max }}$ and $N_{D \text { max }}$ are same as those of $\sigma_{N J \max }$ and $\sigma_{S J \max }$ except that the maximum values of $N_{U \max }$ and $N_{D \max }$ locate at the same point.

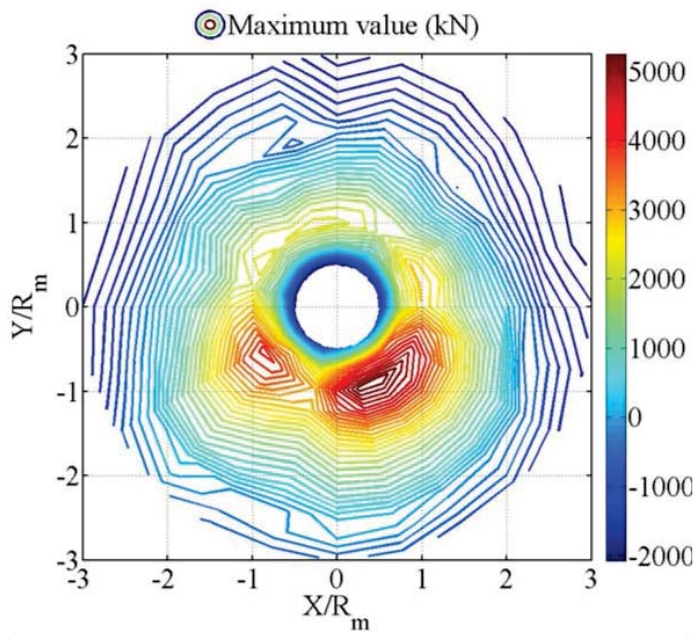

(a) $N_{U \max }$

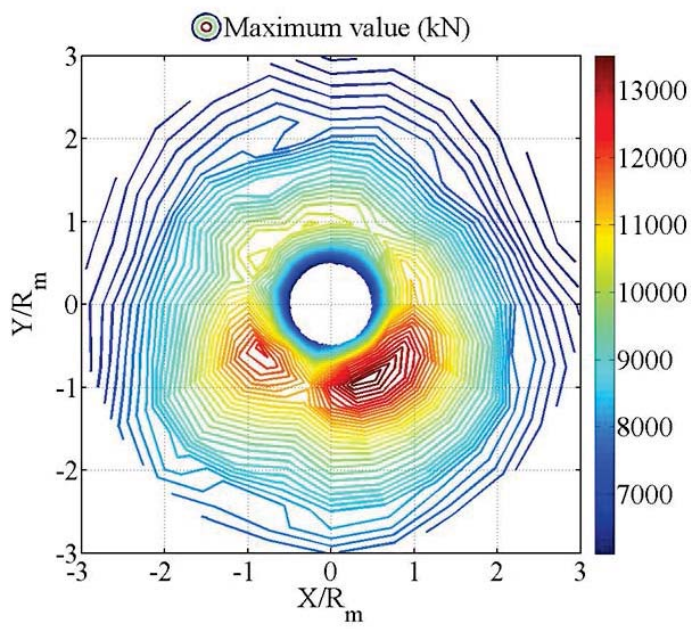

(b) $N_{D \max }$

Figure 14: Contours of maximum uplift axial force and downward axial force on legs

The time history of normalized maximum uplift axial force $N_{U \max }$ and downward axial force $N_{D \max }$ at the points where maximum values occur are shown in Figure 15. From Figure 15 , the time history of normalized maximum uplift axial force $N_{U \max }$ and downward axial force $N_{D \max }$ are bimodal in shape with the maximum values occur before the arrival of mini- 
mum distance to storm center. Except for the specific value, the time history curve of $N_{U \max }$ shows the same trend as that of $N_{U \text { max }}$.

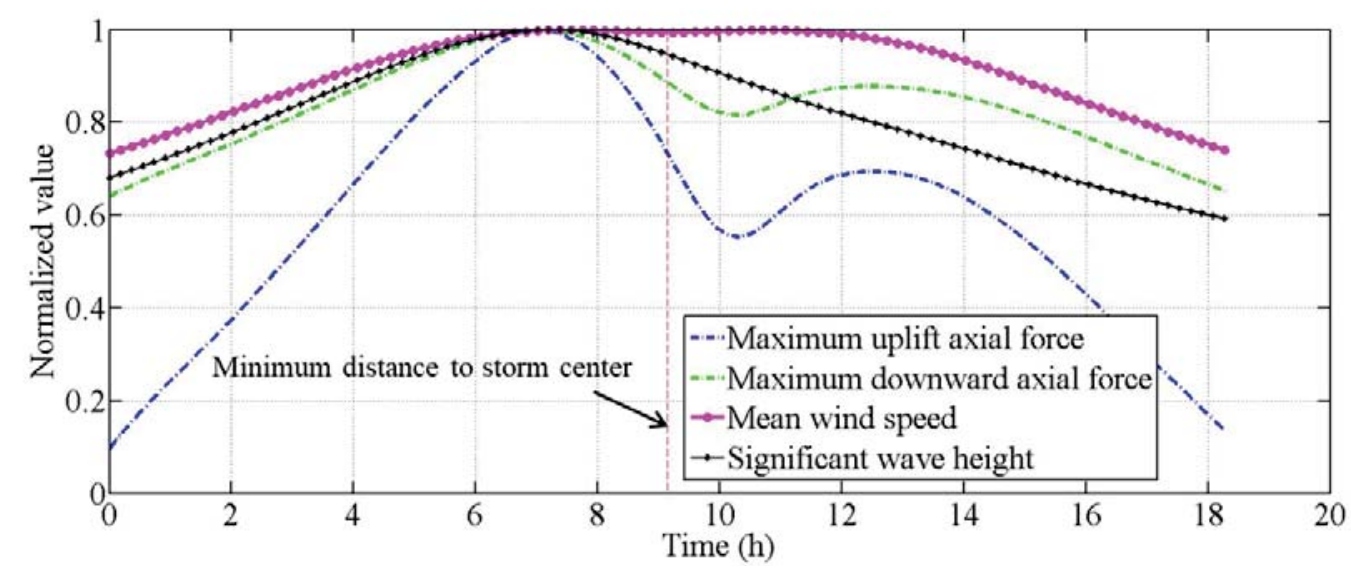

Figure 15: Time history of normalized mean wind speed, significant wave height, maximum uplift axial force and maximum downward axial force

\section{CONCLUSIONS}

In this paper, a series of coupled aero-servo-hydro elastic analyses have been conducted to investigate the spatial distributed characteristics of hurricane induced response of OWTs, by checking the tower-top and tower base displacement, normal stress and shear stress in tower and substructure, uplift and downward axial force of legs, etc. From this study, the following conclusions can be drawn:

(1) As tower-top and platform displacement are dominated by aerodynamic load, the spatial distribution and time history curve of maximum displacement show similar characteristics as mean wind speed. The maximum displacement occurs near the eyewall where the time history curve of maximum displacement shows unimodal characteristic. Moreover, time history curve shows that the maximum displacement occurs before the arrival of minimum distance to storm center.

(2) For structural stress, the spatial distributions of maximum normal stress and shear stress in tower show different characteristics from those in substructure. The spatial distributions of maximum normal stress and shear stress in tower are similar to those of maximum tower-top displacement while the maximum normal stress and shear stress in substructure can be found at the point near the point of maximum significant wave height. Besides, even if the time history curves of mean wind speed and significant wave height are unimodal in shape, the time history curves of normalized maximum normal stress and normalized maximum shear stress are bimodal with the maximum values occur before the arrival of minimum distance to storm center

(3) Being dominantly affected by hydrodynamic load, the spatial distributions of maximum uplift axial force and downward axial force share the same characteristic with those of maximum normal stress and shear stress in substructure. In addition, the time history curves of normalized maximum uplift axial force and downward axial force are bimodal in shape with the maximum values occur before the arrival of minimum distance to storm center. 


\section{REFERENCES}

[1] T. Ishihara, et al, An analysis of damaged wind turbines by Typhoon Maemi in 2003, Sixth Asia-Pacific Conference on Wind Engineering (APCWE VI), 2005.

[2] J. Chou \& W. Tu, Failure analysis and risk management of a collapsed large wind turbine tower, Engineering Failure Analysis 18(1), 295-313, 2011.

[3] Z. Li, et al, Design defect of wind turbine operating in typhoon activity zone, Engineering Failure Analysis 27, 165-172, 2013.

[4] Q. Liu, et al, Numerical simulations of ocean surface waves under hurricane conditions: As-sessment of existing model performance, Ocean Modelling 118, 73-93, 2017.

[5] E. Kim, \& L. Manuel, Hurricane-induced loads on offshore wind turbines with considerations for nacelle yaw and blade pitch control, Wind Engineering 38(4), 413-423, 2014.

[6] J. T. Ta \& G. S. Dwarakish, Numerical wave modelling-A review, Aquatic procedia 4, 443-448, 2015.

[7] G. J. Holland, An analytic model of the wind and pressure profiles in hurricanes, Monthly weather review 108 (8), 1212-1218, 1980.

[8] A. C. Phadke, et al, Modeling of tropical cyclone winds and waves for emergency management, Ocean Engineering 30(4), 553-578, 2003.

[9] H. E. Willoughby \& M. E. Rahn, Parametric representation of the primary hurricane vortex. Part I: Observations and evaluation of the Holland (1980) model, Monthly Weather Review 132 (12), 3033-3048, 2004.

[10] K. Emanuel, et al, A statistical deterministic approach to hurricane risk assessment, Bulletin of the American Meteorological Society 87(3), 299-314, 2006.

[11] N. Lin, Ning \& D. Chavas, On hurricane parametric wind and applications in storm surge modeling, Journal of Geophysical Research: Atmospheres 117(D9), 2012.

[12] I. R. Young \& J. Vinoth, An "extended fetch" model for the spatial distribution of tropical cyclone wind-waves as observed by altimeter, Ocean Engineering 70, 14-24, 2013.

[13] I. R. Young, A review of parametric descriptions of tropical cyclone wind-wave generation, Atmos-phere 8(10), 194, 2017.

[14] I. R. Young \& J. Vinoth, A parametric model for tropical cyclone waves, ASME 2013 32nd International Conference on Ocean, Offshore and Arctic Engineering, American Society of Mechanical Engineers Digital Collection, 2013.

[15] I. J. Moon, et al, Numerical simulation of sea surface directional wave spectra under hurricane wind forcing, Journal of physical oceanography 33(8), 1680-1706, 2003.

[16] P. A. Hwang, Fetch-and duration-limited nature of surface wave growth inside tropical cyclones: With applications to air-sea exchange and remote sensing, Journal of Physical Oceanography 46(1), 41-56, 2016.

[17] P. A. Hwang \& E. J. Walsh, Azimuthal and radial variation of wind-generated surface waves inside tropical cyclones, Journal of Physical Oceanography 46(9), 2605-2621, 2016. 
[18] P. A. Hwang \& Y. Fan, Effective fetch and duration of tropical cyclone wind fields estimated from simultaneous wind and wave measurements: Surface wave and air-sea exchange computation, Journal of Physical Oceanography 47(2), 447-470, 2017.

[19] P. A. Hwang, et al, Ocean surface wave spectra inside tropical cyclones, Journal of Physical Oceanography 47(10), 2393-2417, 2017.

[20] K. Wei, et al, Directional effects on the reliability of non-axisymmetric support structures for offshore wind turbines under extreme wind and wave loadings, Engineering Structures 106, 68-79, 2016.

[21] E. Kim \& L. Manuel, A framework for hurricane risk assessment of offshore wind farms, ASME 2012 31st international conference on ocean, offshore and arctic engineering, American Society of Mechanical Engineers Digital Collection, 2013.

[22] K. Wei, S. R. Arwade \& A. T. Myers, Incremental wind-wave analysis of the structural capacity of offshore wind turbine support structures under extreme loading, Engineering Structures 79, 58-69, 2014.

[23] S. T. Hallowell, et al, Hurricane risk assessment of offshore wind turbines, Renewable energy 125, 234-249, 2018.

[24] W. J. Pierson Jr \& L. Moskowitz, A proposed spectral form for fully developed wind seas based on the similarity theory of SA Kitaigorodskii, Journal of geophysical research 69(24), 5181-5190, 1964.

[25] K. Hasselmann, et al, Measurements of wind-wave growth and swell decay during the Joint North Sea Wave Project (JONSWAP), Ergänzungsheft 8-12, 1973.

[26] C. W. Wright, et al, Hurricane directional wave spectrum spatial variation in the open ocean, Journal of Physical Oceanography 31(8), 2472-2488, 2001.

[27] Y. Fan, et al, Numerical simulations and observations of surface wave fields under an extreme tropical cyclone, Journal of Physical Oceanography 39(9), 2097-2116, 2009.

[28] J. Jonkman, et al. Definition of a 5-MW reference wind turbine for offshore system development, No. NREL/TP-500-38060. National Renewable Energy Lab.(NREL), Golden, CO (United States), 2009.

[29] R. R. Damiani, et al, Assessing the importance of nonlinearities in the development of a substructure model for the wind turbine CAE tool FAST, ASME 2013 32nd International Conference on Ocean, Offshore and Arctic Engineering, American Society of Mechanical Engineers Digital Collection, 2013.

[30] B. J. Jonkman, TurbSim User's Guide v2. 00.00, Natl. Renew. Energy Lab, 2014.

[31] M. J. Tucker \& E. G. Pitt. Waves in ocean engineering. No. Volume 5. 2001.

[32] International Electrotechnical Commission, IEC 61400-3 Wind Turbines Part3: Design Requirements for Off-shore Wind Turbines, International Electrotechnical Commission: Geneva, Switzerland, 2009.

[33] J. R. Morison, J. W. Johnson, \& S. A. Schaaf, The force exerted by surface waves on piles, Journal of Petroleum Technology, 2(05), 149-154, 1950.

[34] J. M. Jonkman \& M. L. Buhl Jr, FAST user's guide, National Renewable Energy Laboratory, Golden, CO, Technical Report No. NREL/EL-500-38230, 2005. 